\title{
Olfactory function distinguishes vascular parkinsonism from Parkinson's disease
}

\author{
R Katzenschlager, J Ziilmans, A Evans, H Watt, A J Lees
}

J Neurol Neurosurg Psychiatry 2004;75:1749-1752. doi: 10.1136/jnnp.2003.035287

Objective: To compare olfactory function in vascular parkinsonism and Parkinson's disease diagnosed according to published clinical diagnostic criteria.

Methods: The University of Pennsylvania smell identification test (UPSIT) was carried out in 14 patients with vascular parkinsonism, 18 with Parkinson's disease, and 27 normal controls matched for age, sex, and smoking status.

Results: UPSIT scores in vascular parkinsonism (mean 26.1, $95 \%$ confidence interval, 23.1 to 29.0$)$ were significantly better than in Parkinson's disease (mean 17.1 (14.5 to 19.7)) $(p<0.0001)$, and did not differ from the healthy controls (mean 27.6 (25.8 to 29.4)) ( $p=0.32$ ).

Conclusions: Testing olfactory function may be helpful in differentiating vascular parkinsonism from Parkinson's disease.

t is now well established that patients with Parkinson's disease have markedly impaired olfactory function. ${ }^{12}$ This clinical feature corresponds to neuropathological findings of Lewy bodies in the anterior olfactory nucleus, showing involvement of the olfactory system in the neurodegenerative process in Parkinson's disease. ${ }^{2}$ Olfactory dysfunction occurs early in the course of the disease ${ }^{34}$ and is independent of "on" or "off" state, treatment, ${ }^{1}$ or age at onset. ${ }^{2}$

Olfactory function has been investigated in other neurodegenerative conditions associated with parkinsonism. It was found to be impaired in dementia with Lewy bodies, ${ }^{5}$ mildly impaired in multiple system atrophy, ${ }^{6}$ and normal in progressive supranuclear palsy, ${ }^{6}$ corticobasal degeneration, ${ }^{6}$ and parkin-positive parkinsonism. ${ }^{7}$

Vascular parkinsonism has been defined neuropathologically as parkinsonism occurring in cerebrovascular disease, after exclusion of Lewy body disease and other neurodegenerative conditions associated with parkinsonism. ${ }^{89}$ However, vascular parkinsonism remains difficult to distinguish clinically from Parkinson's disease, because basal ganglia infarcts can occur without parkinsonism, ${ }^{10}{ }^{11}$ and vascular pathology commonly occurs in Lewy-body Parkinson's disease. ${ }^{12}$ There is evidence from magnetic resonance imaging (MRI) studies suggesting that two different types of vascular lesion may cause vascular parkinsonism: widespread and bilateral ischaemic lesions have been linked to gradual onset parkinsonism, while basal ganglia infarcts have been reported to be associated with acute onset contralateral parkinsonism. $^{13} 14$ This differentiation was confirmed in a recent clinicopathological correlation study. ${ }^{9}$

Our aim in this study was to assess olfactory function in patients with vascular parkinsonism compared with those with Parkinson's disease and normal controls. To increase the accuracy of the clinical diagnosis of vascular parkinsonism, we required the patients to fulfil published clinical diagnostic criteria $^{14}$ and additional stricter clinical and MRI criteria. ${ }^{913}$

\section{METHODS}

Olfactory function was tested using the University of Pennsylvania smell identification test (UPSIT, Sensonics, Haddon Heights, New Jersey, USA). This test kit consists of 40 odours, which are microencapsulated in paper strips and released by scratching with a pencil. Patients are required to make a forced choice from four possible answers for each item, even if no odour is perceived. The maximum score is 40; normal values decrease with age and are lower in men. ${ }^{15}$

Eligible patients had to fulfil published clinical diagnostic criteria, ${ }^{14}$ which include scores for vascular risk factors and for the temporal relation of stroke and onset of parkinsonism. Additionally, the localisation of MRI lesions in relation to the clinical picture, ${ }^{9}$ rather than their absolute number, was taken into account. Relevant cognitive impairment (defined as a score of $\leqslant 24$ on the mini-mental state examination) was an exclusion criterion. L-dopa response was determined, based on the participants' subjective assessment and the case notes, but was not used as an exclusion criterion for vascular parkinsonism.

Consecutive patients attending the movement disorders clinics at the National Hospital for Neurology and Neurosurgery and the Middlesex Hospital, London, who fulfilled the outlined criteria were asked to participate.

Patients with vascular parkinsonism were matched for age, sex, and smoking status with normal controls, who were spouses and carers of patients, and with patients with Parkinson's disease from the same clinics. Parkinson's disease was diagnosed according to the Queen Square Brain Bank criteria. ${ }^{16}$ In Parkinson's disease patients, vascular lesions on MRI precluded participation, the only exception being minimal evidence of small vessel disease in areas other than the basal ganglia, interpreted as normal for age by an independent radiologist. Subjects were excluded if there was a history of nasal or sinus surgery, severe head trauma, obstructive pulmonary disease, or allergies causing nasal congestion.

For statistical analysis, group means were compared using the unpaired $t$ test. The Kolmogorov-Smirnov test for difference from a normal distribution was non-significant, and inspection of histograms revealed no substantial outliers.

\section{RESULTS}

Table 1 shows the demographic and clinical details, imaging results, vascular risk factors, ${ }^{15}$ and UPSIT scores for the 14 patients with vascular parkinsonism. Mean age was 74.1 years in the vascular parkinsonism group, 72.6 years (range 63 to 85 ) in the 27 control subjects, and 70.6 years (64 to 85 ) in the 18 patients with Parkinson's disease. Mean disease duration was 6.6 years for the vascular parkinsonism group and 9.1 years (range 2 to 17) for the Parkinson's disease group. Mean age and disease duration did not differ significantly among the groups.

Abbreviation: UPSIT, University of Pennsylvania smell identification test 


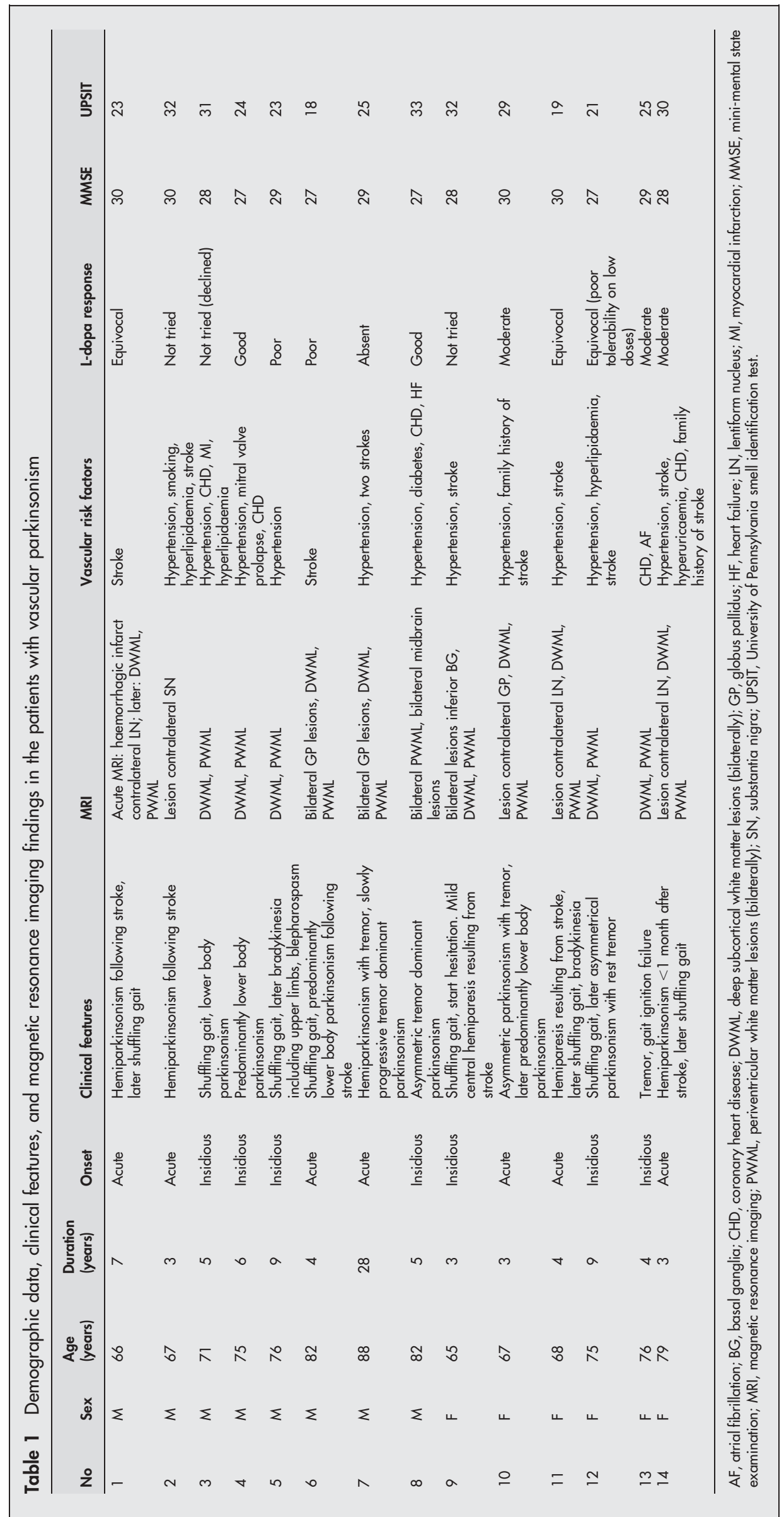




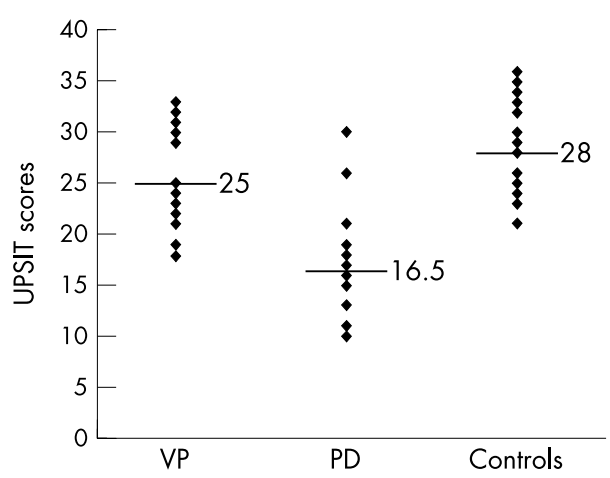

Figure 1 Scatterplot showing UPSIT scores in vascular parkinsonism (VP), Parkinson's disease (PD), and normal controls. Horizontal lines indicate median values. UPSIT, University of Pennsylvania smell identification test.

The mean UPSIT score in the vascular parkinsonism group was 26.1 (95\% confidence interval (CI) 23.1 to 29.0), which was significantly different from the Parkinson's disease group, where mean UPSIT was 17.1 (14.5 to 19.7); $95 \%$ CI of the difference 5.2 to $12.7 ; \mathrm{p}<0.0001)$. Mean UPSIT in the normal controls was 27.6 (25.6 to 29.4). Median values are shown in fig 1 . The difference between Parkinson's disease and controls was significant (95\% CI of difference, -13.5 to $-7.6 ; \mathrm{p}<0.0001)$, while the difference between vascular parkinsonism and controls was non-significant $(p=0.32)$. In this elderly age group, an UPSIT score of $>22$ had a sensitivity of $85.7 \%$ for detecting vascular parkinsonism and a specificity of $88.9 \%$ for distinguishing vascular parkinsonism from Parkinson's disease. As the subjects' age distribution spanned ages where considerable changes in olfactory function normally occur, ${ }^{15}$ we analysed separate cut off points for two age groups (65-75 and 76-88 years). The cut off value showing the best balance between sensitivity and specificity was $\leqslant 23$ in the $65-75$ group, with $100 \%$ sensitivity and a specificity of $85.7 \%$. In the $76-88$ group, an UPSIT score of $\leqslant 22$ yielded a sensitivity of $85.7 \%$ and a specificity of $80 \%$.

\section{DISCUSSION}

Our results show that olfactory function in patients with a clinical diagnosis of vascular parkinsonism is substantially better than in patients with probable Parkinson's disease in whom relevant vascular lesions have been excluded. Olfactory function in vascular parkinsonism did not differ significantly from that in age matched normal controls. We conclude from our findings that testing patients' sense of smell may be a useful adjunct for differentiating between Parkinson's disease and suspected vascular parkinsonism.

The UPSIT can be done quickly and easily in an outpatient setting and can be self administered by patients. However, in view of possible cultural factors influencing results, normal values as published may not be universally applicable and may need to be established for each centre. It may therefore be of interest to investigate alternative testing methods in this patient population. Precise age matching is important in a group of patients where age has a considerable impact on olfactory performance.

The clinical presentation of cerebrovascular disease may be indistinguishable from Parkinson's disease. In a clinicopathological study of 100 patients with a clinical diagnosis of Parkinson's disease, neuropathological examination confirmed this diagnosis in only $76 \% .^{17}$ In three of the 24 misdiagnosed patients, cerebrovascular disease was the only abnormality. A recent clinicopathological study of vascular parkinsonism found that nine of 17 patients had been diagnosed as having Parkinson's disease during life, ${ }^{9}$ also reflecting difficulties in differential diagnosis which exist in clinical practice.

The concept of vascular parkinsonism as a clinical entity was proposed in $1929 .{ }^{8}$ Traditionally, "lower body parkinsonism"18 with predominant lower limb involvement and gait ignition failure was attributed to a vascular aetiology, ${ }^{19}$ while features such as asymmetrical onset, tremor, upper limb involvement, and good L-dopa response were believed to represent Parkinson's disease. Recent clinical-radiological studies in suspected vascular parkinsonism have indicated that cerebrovascular disease can cause a variety of parkinsonian features ${ }^{813}{ }^{14}$ and a clinicopathological study showed a relation between the clinical presentation and the localisation and type of vascular lesions. ${ }^{9}$

Although most studies using psychophysical olfactory tests in Parkinson's disease showed little correlation with disease duration, ${ }^{1}$ there is evidence from olfactory evoked responses suggesting some impact of progressing disability on olfactory function. ${ }^{20}$ The early involvement of olfactory neurones in the neurodegenerative process in Parkinson's disease ${ }^{21}$ explains the findings of early, including presymptomatic, smell deficits. ${ }^{4}$ This suggests that olfactory testing may help in the differential diagnosis of Parkinson's disease and vascular parkinsonism when applied early.

A limitation of our study is the fact that the applied diagnostic criteria for vascular parkinsonism have not yet been validated prospectively. However, by requiring fulfilment of two sets of diagnostic criteria, we attempted to reduce the number of patients with alternative diagnoses. It is possible that excluding L-dopa responsive patients might have further increased the diagnostic accuracy for vascular parkinsonism. However, some degree of L-dopa responsiveness has been found in $23-71 \%$ of pathologically confirmed vascular parkinsonism cases, ${ }^{13} 2223$ and motor complications may occur. ${ }^{23}$

As would be expected, we found a small overlap in UPSIT scores between the groups: Four $(28.6 \%)$ of the 14 patients with vascular parkinsonism and seven $(25.9 \%)$ of the 27 controls had scores below published optimal cut off values for the respective age and sex groups in an American population. ${ }^{24}$ This most probably reflects the fact that these reference values do not distinguish age groups above 71 and therefore do not fully represent the continuing olfactory loss in very elderly subjects, such as those involved in this study. Moreover, it has been suggested ${ }^{26}{ }^{25}$ that the UPSIT normative values may not be easily transferable from the USA to other countries, where several of the odours used in the test are not familiar. It remains a possibility that some of the hyposmic vascular parkinsonism patients had subclinical or co-morbid Lewy body changes in addition to cerebrovascular disease.

The proportion of normal scores that we found in the patients with Parkinson's disease and the sensitivity of UPSIT for distinguishing between Parkinson's disease and vascular parkinsonism are in keeping with most reports showing olfactory dysfunction in $70-90 \%$ of Parkinson's disease patients. ${ }^{12}$

We conclude that, in contrast to Parkinson's disease, the majority of patients with vascular parkinsonism and without associated dementia have a preserved sense of smell. Testing olfactory function may help in the differential diagnosis between two important causes of parkinsonism in the elderly.

\footnotetext{
Authors' affiliations

R Katzenschlager, A J Lees, Reta Lila Weston Institute of Neurological Studies, London W1, UK

A Evans, National Hospital for Neurology and Neurosurgery, Queen
} Square, London WCl 
J Zijlmans, Vrije Universiteit Medisch Centrum, Amsterdam, Netherlands H Watt, Medical Statistics Unit, London School of Hygiene and Tropical Medicine and Institute of Neurology, Queen Square, London WC1

Competing interests: none declared

Correspondence to: Professor A J Lees, Reta Lila Weston Institute of Neurological Studies, Windeyer Building, 46 Cleveland Street, London WIT 4JF, UK; alees@ion.ucl.ac.uk

Received 30 December 2003

In revised form 16 February 2004

Accepted 7 March 2004

\section{REFERENCES}

1 Doty RL, Deems DA, Stellar S. Olfactory dysfunction in parkinsonism: a general deficit unrelated to neurologic signs, disease stage or disease duration. Neurology 1988;38:1237-44.

2 Hawkes $\mathrm{CH}$. Olfaction in neurodegenerative disorder. Mov Disord 2003; 18:364-72

3 Tissingh G, Berendse HW, Bergmans P, et al. Loss of olfaction in de novo and treated Parkinson's disease: possible implications for early diagnosis. Mov Disord 2001;16:41-6.

4 Berendse HW, Booij J, Francot CM, et al. Subclinical dopaminergic dysfunction in asymptomatic Parkinson's disease patients' relatives with a decreased sense of smell. Ann Neurol 2001;50:34-41.

5 Liberini P, Parola S, Spano PF, et al. Olfactory dysfunction in dementia associated with Lewy bodies. Parkinsonism Rel Disord 1999;5:30.

6 Wenning GK, Shephard B, Hawkes C, et al. Olfactory function in atypical parkinsonian syndromes. Acta Neurol Scand 1995:91:247-50.

7 Khan NL, Katzenschlager R, Watt H, et al. Olfaction differentiates parkin disease from early-onset parkinsonism and Parkinson disease. Neurology 2004;62:1224-6.

8 Critchley M. Arteriosclerotic parkinsonism. Brain 1929;52:23-83.

9 Zijlmans JCM, Daniel SE, Hughes AJ, et al. A clinico-pathological investigation of vascular parkinsonism (VP), including clinical criteria for the diagnosis of VP. Mov Disord (Early view): www3.interscience.wiley.com/cgi bin/fulltext/017638030/HTMLSTART
10 Weiller C, Ringelstein EB, Reiche W, et al. The large striatocapsular infarct. A clinical and pathophysiological entity. Arch Neurol 1990;47:1085-91.

11 Van Zagten M, Lodder J, Kessels F. Gait disorder and parkinsonian signs in patients with stroke related to small deep infarcts and white matter lesions. Mov Disord 1998;13:89-95.

12 Hughes AJ, Daniel SE, Blankson S, et al. A clinicopathologic study of 100 cases of Parkinson's disease. Arch Neurol 1993;50:140-8.

13 Zijlmans JCM, Thijssen HOM, Vogels OJM, et al. MRI in patients with suspected vascular parkinsonism. Neurology 1995;45:2183-8.

14 Winikates J, Jankovic J. Clinical correlates of vascular parkinsonism. Arch Neurol 1999;56:98-102.

15 Doty RL, Shaman P, Dann M. Development of the University of Pennsylvania Smell Identification Test: a standardized microencapsulated test of olfactory function. Physiol Behav 1984;32:489-502.

16 Gibb WRG, Lees AJ. The relevance of the Lewy body to the pathogenesis of idiopathic Parkinson's disease. J Neurol Neurosurg Psychiatry 1988;51:745-52

17 Hughes AJ, Daniel SE, Kilford L, et al. Accuracy of clinical diagnosis of idiopathic Parkinson's disease: a clinico-pathological study in 100 cases. J Neurol Neurosurg Psychiatry 1992;55:181-4.

18 FitzGerald PM, Jankovic J. Lower body parkinsonism: evidence for a vascular etiology. Mov Disord 1989;4:249-60.

19 Thompson PD, Marsden CD. Gait disorder of subcortical arteriosclerotic encephalopathy: Binswanger's disease. Mov Disord 1987;2:1-8.

20 Hummel T. Olfactory evoked potentials as a tool to measure progression of Parkinson's disease. In: Chase T, Bedard B, eds. Focus on medicine, vol 14 New developments in the drug therapy of Parkinson's disease. Oxford: Blackwell Science, 47-53.

21 Braak H, Rüb U, Gai WP, et al. Idiopathic Parkinson's disease: possible routes by which vulnerable neuronal types may be subject to neuroinvasion by an unknown pathogen. J Neural Transm 2003;110:517-36.

22 Demirkiran M, Bozdemir H, Sarica Y. Vascular parkinsonism: a distinct, heterogeneous clinical entity. Acta Neurol Scand 2001;104:63-7.

23 Ziilmans JCM, Katzenschlager R, Daniel SE, et al. The L-dopa response in vascular parkinsonism. J Neurol Neurosurg Psychiatry 2004;75:545-7.

24 Doty RL, Bromley SM, Stern MB. Olfactory testing as an aid in the diagnosis of Parkinson's disease: development of optimal discrimination criteria. Neurodegeneration 1995;4:93-7.

25 Gray AJ, Staples V, Murren K, et al. Olfactory identification is impaired in clinic-based patients with vascular dementia and senile dementia of Alzheimer type. Int J Geriatr Psychiatry 2001;16:513-17. 